\title{
Miocardiopatía periparto con trombosis biventricular
}

\author{
J. SÁNCHEZ-RUBIO LEZCANO, J. G. GALACHE OSUNA, A. MARQUINA \\ BARCOS, I. CALVO CEBOLLERO, J. A. DIARTE DE MIGUEL, L. J. PLACER \\ PERALTA
}

Servicio de Cardiología. Hospital Universitario Miguel Servet. Zaragoza

\author{
PERIPARTUM CARDIOMYOPATHY WITH BIVENTRICULAR \\ THROMBI
}

\section{RESUMEN}

La miocardiopatía periparto es una causa poco frecuente de insuficiencia cardiaca congestiva que se desarrolla durante el último mes de embarazo o en los 5 primeros meses del puerperio en mujeres sin cardiopatía previa conocida. La presencia de trombosis intraventricular no es infrecuente y puede ser identificada mediante ecocardiografía. Presentamos el caso de una paciente afecta de miocardiopatía periparto en la cual el ecocardiograma demostró la presencia de trombosis biventricular, hallazgo excepcional dado que sólo se recogen otros 2 casos en la literatura. Comentamos brevemente el diagnóstico y tratamiento de esta patología y las circunstancias especiales que pueden favorecer la formación de trombos en estas pacientes.

PALABRAS CLAVE: Miocardiopatía periparto. Trombosis. Tratamiento.

\begin{abstract}
Peripartum cardiomyopathy is a rare cause of congestive heart failure which developes in the last month of pregnancy or during five months postpartum in women without previously known cardiac disease. Intraventricular thrombi are no unusual and can be identified by echocardiography. We report the case of a woman with peripartum cardiomyopathy whose echocardiographic study showed the presence of biventricular thrombi. This is a quite rare finding with only two cases reported in the literature. Diagnosis, treatment and special situations that could predispose to intracardiac thrombi formation are discussed.
\end{abstract}

KEY WORDS: Peripartum cardiomyopathy. Thrombosis. Treatment.

Sánchez-Rubio Lezcano J, Galache Osuna JG, Marquina Barcos A, Calvo Cebollero I, Diarte de Miguel JS, Placer Peralta LJ. Miocardiopatía periparto con trombosis biventricular. An Med Interna (Madrid) 2004; 21: 498-500.

\section{INTRODUCCIÓN}

En 1971 Demakis y cols. definen la miocardiopatía periparto (MP) como el desarrollo de disfunción sistólica del ventrículo izquierdo durante el último mes de embarazo o en los 5 primeros meses del puerperio, sin una causa identificable y en una paciente sin enfermedad cardiaca previa conocida (1). La presencia de trombos intracardiacos en el contexto de MP es mayor que en otras formas de miocardiopatía dilatada. Se trata de un hallazgo relativamente frecuente y han sido identificados en series necropsicas y mediante ecocardiografía (2).

\section{CASO APORTADO}

Paciente de 39 años sin antecedentes de interés que tras su cuarto parto eutócico desarrolla insuficiencia cardiaca. En ese momento se realizó un ecocardiograma que demostró un ventrículo izquierdo dilatado con fracción de eyección del $28 \%$ y sin otros hallazgos, diagnosticándose MP que fue tratada de manera convencional asociando acenodicumarol. Tras 6 meses de evolución reingresa por cuadro de insuficiencia cardiaca aguda. Los resultados de las pruebas complementarias figuran en la tabla I. El ecocardiograma practicado al ingreso mostraba un ventrículo izquierdo dilatado, de morfología esferoidal, con severa depresión global de la contractilidad $($ FEVI $<20 \%$ ) (Fig. 1A). En el plano apical modificado se visualizan dos masas adheridas al septo medio y basal de ventrículo izquierdo que sugieren trombos (Fig. 1B) y se identifican nuevamente en el plano paraesternal eje corto (Fig. 2A). En el plano subcostal (Fig. 2B) se observa con mayor definición la presencia de un gran trombo ocupando el apex de ventrículo derecho y otro trombo adherido al septo de ventrículo izquierdo que alcanza el apex.

Se trata de una paciente con bajo nivel sociocultural y una baja adhesión al tratamiento. No tomaba con regularidad el acenodicuma-

Trabajo aceptado: 20 de abril de 2004

Correspondencia: Juan Sánchez-Rubio Lezcano. Servicio de Cardiología. Hospital Universitario Miguel Servet. Paseo Isabel la Católica, 1-3. 50009 Zaragoza. e-mail: srljuan@terra.es. 
TABLA I

\section{PRUEBAS COMPLEMENTARIAS}

Radiografía de tórax: cardiomegalia global, signos de congestión venosa pulmonar

ECG: taquicardia sinusal a $105 x^{\prime}$

Bioquímica: Na $132 \mathrm{mEq} / \mathrm{l}, \mathrm{K}$ 3,6 mEq/l, Cl $98 \mathrm{mEq} / \mathrm{l}$, urea $42 \mathrm{mg} / \mathrm{dl}$, creatinina $1,2 \mathrm{mg} / \mathrm{dl}$, glucosa $118 \mathrm{mg} / \mathrm{dl}$

Hemograma: hematíes $4.6 \times 10^{6} / \mathrm{mm}^{3}$, leucocitos $7,5 \times$ $10^{3} / \mathrm{mm} 3$, plaquetas $284 \times 10^{3} / \mathrm{mm}^{3}$

Coagulación: TTP 32", T. Quick 13,3", actividad de protombina $78 \%$, INR 1,3
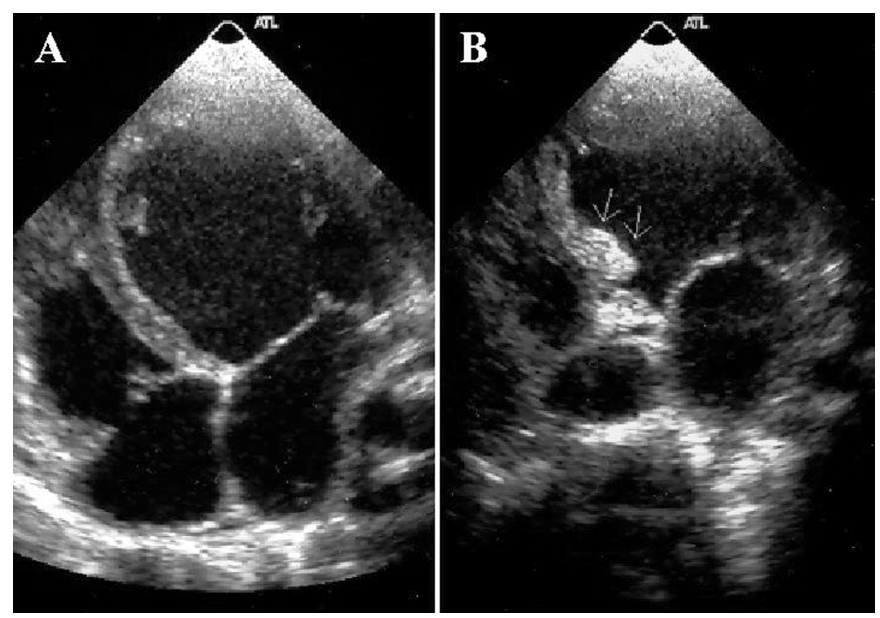

Fig. 1.
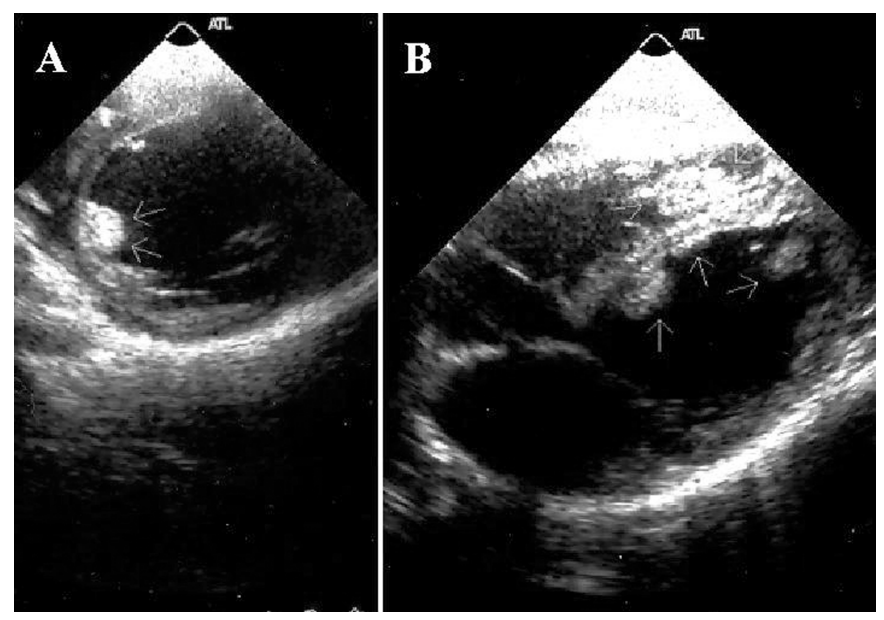

Fig. 2.

rol que tenía prescrito y presentaba una actividad de protrombina del $78 \%$, aspecto que seguramente resultó determinante en la formación de los trombos intracardiacos. Se instauró tratamiento con heparina sódica intravenosa y se continuó posteriormente con acenodicumarol vía oral, comprobándose en un ecocardiograma de control la desaparición de las masas, punto este que confirma el diagnóstico de trombosis biventricular. La paciente respondió de manera adecuada a la terapia intravenosa con inótropos y diuréticos siendo dada de alta pendiente de estudio hemodinámico dentro del protocolo de valoración como candidata a transplante cardiaco.

\section{DISCUSIÓN}

El diagnóstico de la MP se basa en los criterios propuestos por Demakis y cols. (1) y actualizados en 1997 por los participantes del Nacional Heart, Lung, and Blood Institute Workshop. Así, se entiende por MP al desarrollo de un cuadro de insuficiencia cardiaca durante el último mes de embarazo o en los primeros 5 meses de puerperio sin una causa identificable, en ausencia de enfermedad cardiaca conocida antes del último mes de embarazo. La ecocardiografía es una herramienta fundamental para establecer el diagnóstico y es requisito indispensable la demostración de disfunción sistólica del ventrículo izquierdo $(3,4)$.

La incidencia real de esta enfermedad es difícil de estimar y las cifras publicadas presentan una alta variabilidad, pero podría establecerse entre 1/3000 y 1/4000 (5-7). La raza negra, la multiparidad, los embarazos gemelares, le edad mayor de 30 años, los antecedentes familiares, la preclamsia/eclamsia y la hipertensión gestacional se han relacionado con una mayor probabilidad de padecer MP (8).

La etiología de la MP continúa siendo una incógnita. Se han propuesto varias hipótesis como una inadecuada adaptación del corazón al estrés hemodinámico del embarazo, factores nutricionales, endocrinos, etc., pero probablemente sea debida al desarrollo de una miocarditis de origen infecciosa o autoinmune en pacientes genéticamente predispuestas $(5,9)$.

El tratamiento de la MP no difiere del de otras formas de miocardiopatía dilatada idiopática, aunque si el cuadro se desencadena durante el embarazo, deberemos evitar fármacos nocivos para el feto como los IECA, que podrán ser sustituidos por otros vasodilatadores como el amlodipino o nitratos más hidralacina (Tabla II). En casos de evolución tórpida con insuficiencia cardiaca refractaria se ha realizado trasplante cardicaco con resultados similares a otras formas de miocardiopatía dilatada (10).

Los fenómenos tromboembólicos son frecuentes en esta patología y se han publicado varios casos de trombosis intracardiaca diagnosticada por ecocardiografía. Sin embargo lo

\section{TABLA II}

MEDIDAS Y FÁRMACOS ÚTILES EN EL TRATAMIENTO DE LA MIOCARDIOPATÍA PERIPARTO

\begin{tabular}{ll}
\hline Tratamiento de la miocardiopatía periparto \\
\hline Antes del parto & Después del parto \\
\hline Reducción hidrosalina & Reducción hidrosalina \\
$\mathrm{O}_{2}$ & $\mathrm{O}_{2}$ \\
Reposo relativo & Reposo relativo \\
Diuréticos & Diuréticos \\
Digoxina & Digoxina \\
Betabloqueantes & Betabloqueantes \\
Heparina & Anticoagulantes orales \\
Amlodipino & IECA \\
Nitratos más hidralazina & ARA II \\
\hline
\end{tabular}


extraordinario de este caso es la presencia de trombos intracavitarios en ambos ventrículos dado que solo tenemos constancia de dos casos de trombosis biventricular recogidos en la literatura $(2,12)$.

La facilidad con la que las afectas de MP desarrollan trombos intraventriculares es debida a varios factores. Por un lado, de igual forma que en otras miocardiopatías dilatadas, la depresión de la fracción de eyección y la dilatación de las cavidades cardiacas supone un estasis sanguíneo que favorece la formación de trombos. Por otro lado, el embarazo constituye por si mismo un estado procoagulativo debido al aumento de los niveles plasmáticos de los factores VII, VIII, X y del fibrinógeno, así como de la agregabilidad plaquetaria, situación que se mantiene al menos hasta la sexta semana postparto (13). Por ello, estas pacientes se benefician de tratamiento crónico con dicumarínicos (heparina durante la gestación) para la prevención de fenómenos cardioembólicos, que deberá mantenerse hasta la desaparición de los trombos intracavitarios si los hubiere y siempre que exista dilatación de cavidades y disfunción sistólica $(5,11)$. Esta es la actitud que adoptamos en esta paciente y tras 6 meses de seguimiento no ha padecido ningún episodio de embolia periférica o pulmonar, ni se han documentado nuevas masas intracavitarias en los ecocardiogramas de control.

\section{Bibliografía}

1. Demakis JC, Rahimtola SH. Peripartum cardiomyopathy. Circulation 1971; 44: 964-968.

2. Nishi I, Ishimitsu T, Ishizu I, Ueno Y, Suzuki A, Seo Y, et al. Peripartum cardiomyopathy and biventricular thrombi. Circ J 2002; 66: 863865.

3. Pearson GD, Veille JC, Rahimtoola S, et al. Peripartum cardiomyopathy: National Heart, Lung and Blood Institute and Office of Rare Diseases (National Institutes of Health) workshop recommendations and review. JAMA 2000; 283: 1183-1188.

4. Hibbard JU, Lindheimer M, Lang RM. A modified definition for peripartum cardiomyopathy and prognosis based on echocardiography. Obstet Gynecol 1999; 94: 311-316.

5. Ruiz M, Lopez A, Fierro LJ. Miocardiopatía periparto. Med Clin (Barc) 2000; 114: 551-557.

6. Ventura SJ, Peters KD, Martin JA, Maurer JD. Births and deaths: United States, 1996. Mon Vital Stat Rep 1997; 46: (Supl. 2).

7. Cunningham FG, Pritchard JA, Hankins GDV. Peripartum heart failure:

idiopathic cardiomyopathy or compounding cardiovascular events? Obstet Gynecol 1986; 67: 157-68.

8. Mehta NJ, Mehta RN, Khan IA. Peripartum cardiomyopathy: clinical and therapeutic aspects. Angiology 2001; 52 (11): 759-62.

9. Ansari AA, Fett JD, Carraway RE, Mayne AE, Onlamoon N, Sundstrom JB. Autoinmune mechanisms as the basis for human peripartum cardiomyopathy. Clin Rev Allergy Inmunol 2002; 23 (3): 301-24.

10. Christopher S, Brown MD, Barry D, Bertolet MD. Peripartum cardiomyopathy: A comprehensive review. Am J Obstet Gynecol 1998; 409-14.

11. Mehta NJ, Mehta RN, Khan IA. Peripartum cardiomyopathy: clinical and therapeutic aspects. Angiology 2001; 52 (11): 759-62.

12. Bhat PS, Kattakkayam T, Shah RK, Reddy MM, Narayanan GR. Peripartal cardiomyopathy with biventricular thrombi presenting as acute saddle embolism: a case report. Indian Heart J 1986; 38: 486-488.

13. Lampert MB, Lang RB. Peripartum Cardiomiopathy. Am Heart J 1995; 130: 860-870. 